\title{
Theoretical Models, Case Studies and Simulation Games as Knowledge and Decision Tools
}

The methodological challenges identified by this book reflect the limitations of or the constraints on the ontological conceptualization of transformation towards sustainability due to complexity and uncertainty. This complexity is exacerbated by the inevitable contextualization of sustainable, low-carbon transformation, where there is the need to find ways to assess and understand the political, economic, cultural, technological and environmental context from many analytical and governance levels, degrees and scales of causalities as well as making use of insights from these contexts to draw up lessons for others. The context, as reflected by socio-technical and socio-linguistic narratives, not only defines power relations between agents (e.g., between change agents and status quo agents), but also constitutes the audience as codified by social contracts.

The historicity challenge of sustainable low carbon development is driven by the inherent connection of the past to the present and future. Because historical events are usually analyzed in retrospect and as 'finished products,' the empirical value of historical analyses is defined by what has already happened. For example, the life-course analysis focuses on the overlaps between individual experiences and those coincident in history within specific structural, social and social contexts (e.g., changes in government policy) (see Elder et al. 2003; White \& Klein 2007; Mayer 2009). As such, questions that are formulated in historical analyses tend to depict closed processes.

As the theory of path dependence implies, decisions made in the past limit the set of possible present and future decisions (see David 1988; Arthur 1994). Therefore, analyzing the historical components of current and future policies, governance structures and human behavior can only be useful for the analysis of transformation processes when the relevance of these historical components to the present and the future can be established and understood. However, as it will 
be evident in the case study chapters in this book, arguments of historical inevitability based on formulating examples of historical contingency of how different choices could have led to different consequences may be misleading, because of the inability to test the occurrence of 'alternative paths'. The comparison of a 'real' development with its version in an 'alternate universe' has limited empirical value due to its highly speculative character. Therefore, due to the historicity of case studies, interpretations of cause-and-effect relations can only be tentative, and they need to be regularly revisited and corrected.

While 'learning from history' is intuitively accepted, there are limitations to the significance of history for studying the present and the 'futures,' primarily because the 'formalization' of historical analyses constantly needs further development. Charles Tilly (1987) identifies deficits in formalization in historical analysis as important factors in understanding the limitations of the value of historical analyses. Formalization requires the conceptualization, measurement, modelling, and estimation of historical events. However, as Charles Tilly (1987 p. 20) further claims, formalizing historians have devoted little of their ingenuity to the statement of an historical question as a problem susceptible to a formal statement (conceptualization), to the organization of evidence in a standard, comparable form (measurement), to the formal statement of an argument concerning the expected pattern of a phenomenon (modelling), and to matching models to evidence in order to see how the model fits (estimation).

Scholars working on sustainable, low-carbon transformation will need to ensure such formalization, which will very likely require multi- and interdisciplinary approaches and expertise. For example, conceptualization is likely to be conducted by social scientists with comprehensive and integrated knowledge that would allow the establishment and validation of concepts, labels, terminologies, and notions. Conceptualization needs to identify and justify the boundaries and scopes of the subjects of the analyses. It will also need to accommodate competing historical narratives. In addition, measurement and modelling will require scholars with expertise to identify and filter relevant data and information. These scholars should be able to simplify a complex system without losing empirical relevance. Additional scholars are needed to calibrate models and connect them to empirical reality to make them useful for decision-making. The assessment of the value of these models is often defined by how they can help provide policy-makers with the capacity to justify decisions.

Also relevant to the methodological challenge brought by path dependent historicity is the plurality of historical explanations due to limitations on accessing all relevant information. As many of the decisions were made in closed-door meetings, the assessment (process-tracing) of these historical events is confronted 
by a plurality of possible explanations. These explanations can be regarded as storylines that are composed of arguments supporting cause-and-effect relationships. This plurality is likely to be reproduced when establishing narratives and enduring issues.

In addition, analyzing the 'Gestaltungsmacht' (shaping power) of historical events vis-à-vis the future is a contentious endeavor, particularly because of the need to clearly identify and trace the cause-and-effect relationships between factors and monitor the long-term developments that transcend spatial and temporal boundaries. Moreover, not only are there many ways of 'packing' a concert of historical events, there are also latent self-driving processes that can only be identified in retrospect, thus limiting the ability to predict the future. Another concern is that, particularly in sustainable, low-carbon transformations, there is not only one future caused by various possible concerts of historical events, but many futures that can be possibly caused by one or more concerts of historical events. Inserting the value of perspectives in the analysis brings the challenge that one future, say the sustainable, low-carbon transformation, cannot, under current conditions, be optimal for several actors, because they are confronted with other points of departure. As there can be more than one optimal future scenario, collaboration and coordination problems need to be resolved as forethoughts.

\subsection{Scenarios as Methods of Analysis of Futures}

The scientific literature on climate mitigation and low-carbon transformation relies heavily on the use of scenario methods. The complexity as well the temporal dimension of relevant processes and outcomes require decision-makers to use decision tools to structure decision-making. For example, the reduction of emissions will require years if not decades before substantial effects will be significant. As such, present decision-makers and their constituents will most likely not personally experience the outcomes of their decisions. Similarly, some positive effects might take place in other areas leading to these effects becoming intangible for those who would need to accept present burdens of actions. Nevertheless, as it is assumed that delaying decisions or even avoiding making decisions will lead to more 'painful' and, therefore, unacceptable costs, decisions are still to be made. Because decision outcomes can be theoretical at best, while actions are practical, tools are needed to bridge the gaps between these two analytical levels. Inevitably, decision-makers are required to formulate visions or goals that would frame decisions, or even set or change paradigms. Goals have a temporal dimension as they imply achieving a specific status in the future. Therefore, 
formulating policy goals will require tools that address the need to 'prepare' for the future.

Scenarios are constructed to investigate alternative future developments under a set of assumed or controlled conditions. Scenarios are instruments to help decision-makers cope with problem cases that are complex and uncertain. As Nick Hughes (2009) observes, recent years have seen a proliferation in the use of scenario methods to define low-carbon futures. 'Energy in a Finite World' of the International Institute of Applied Systems Analysis is one of the first global studies that have used scenarios as a tool to assess future $\mathrm{CO}_{2}$ emissions in the late 1970s (Häfele et al. 1981). The IPCC has used emissions and climate scenarios as a central component of its work as an institution that assesses climate change research. Working with authors and modelers, it has developed emission scenarios to be used for driving global circulation models to develop climate scenarios. The SA90 scenarios were introduced by Working Group 1 of the IPCC in 1990 (IPCC 1990).

Some questions that a scenario developer needs to address include how scenarios can provide insights not only into the integrated scope or description of the system being studied, but also how the system may have evolved into its current state and more importantly, how the system might continue to evolve, taking into consideration changing motivations and functionalities. What are the possible systemic effects of changes in the motivation and behavior of actors? This will most likely invoke the re-evaluation of various elements of the scenario, asking whether and how the actors could have decided to resolve these changes in a different manner. Asking these questions implies that scenarios have a normative undertone.

There are limitations on decisions based on scenarios. Scenarios often do not envisage changes in an actor's preferences, as well as in relations between actors. Therefore, scenarios are often limited to providing insights on behavioral change and paradigm shifts that are highly valuable in any analysis of transformation processes. Because choices will depend on temporal and spatial circumstances, humans can change their decisions when circumstances change. As Michel Godet (2000) argues, humans can be both rational and driven by the emotional faculties. While scenarios are often not able to capture these changes, they are also often designed assuming the rationality of decisions and actions. In addition, as circumstances change, human interactions also change. Iterative actor interactions imply changing coalitions, behavior and identities. Scenarios, therefore, need to be complemented by tools that support the anticipation of changes in an actor's preferences and relationships.

Furthermore, scenarios are often not able to properly address the dynamics of social, technological, and economic change. As social, technological, and 
economic changes unfold, new issues may arise, or old issues may gain a different meaning and significance, prompting the need to revise previous decisions. For example, the dynamics of change may imply a new importance on certain conflict cleavages that will touch on differences between actors in terms of their values and even ideologies. Because of this, the agreements and other outcomes of previously conducted bargaining games will require renegotiation and re-elaboration. In some cases, renegotiations will need to start by default. In other cases, particularly when the procedures of renegotiations were included in previous agreements, bargaining over new issues will be met by amendments or additional protocols to existing agreements.

One scenario is a collection of conditions and factors that jointly express a specific storyline. The storyline is heuristic in the sense that it employs an approach to problem-solving that does not guarantee or pursue 'optimality.' A scenario is an analytical instrument through which assumptions or 'stories' are made about the system under the conditions of limited knowledge and information. Therefore, a scenario inevitably pertains to a deviation from the 'optimal solution', as optimality presumes complete information. Nevertheless, it is not the objective of scenarios to present the optimal solution or the 'perfect story', but rather to look for a simple set of conditions sufficient to achieve immediate goals. This storyline serves as the structure in decision-making, limiting the number of possible decisions, thus, reducing indecisiveness. For example, the storyline of a scenario may highlight the necessity to adhere to human rights in present and future actions. It encourages decisions and actions by providing the scope of necessary actions and eliminating possible decisions and actions that do not correspond with the storyline. Nevertheless, the storyline, which is often a result of consensus-building processes, needs, as a normative approach, to be inclusive and should support the orchestration of various negotiation processes.

\subsection{Theoretical Models and the 'Ideal Type'-A New- Old Method of Analysis}

An ideal type can be regarded as a distinct type of model or scenario. One major difference between an ideal type and a model or scenario lies in its methodological implication. When a model or scenario depicts a historical or trajectory-based process, as is the case with the low-carbon transformation of energy systems, an understanding of purposes and the related causal interdependencies becomes necessary. Can properly identified indicators be adequately measured and interpreted? Are the benchmarks being fulfilled in order to achieve the predetermined 
purposes as the process unfolds? Is there room for tolerance and the adjustment of targets? Are there relevant self-driving dynamics that need to be addressed? The usage of ideal types allows researchers to focus on the individuality of actors, structures, issues, processes, and outcomes, without undermining the scientific validity of the results of analyses. It allows the 'systematic' management of indicators and benchmarks.

The 'ideal type' is a typological term most closely associated with the sociologist Max Weber (1864-1920), in which Weber claimed a better understanding (Verstehen) of historical development through ideal types (Weber 1976, 1985). Georg Simmel (1858 to 1918), a contemporary of Weber's, has adapted Weber's line of thought and developed a similar term called "categories," which are ideal types that not only serve as principles for constructing terms and concepts but as sources of theoretical knowledge (see Simmel 1908).

Similar to scenarios, ideal types can be identified as analytical instruments designed to strategically facilitate decision-making. Ideal types formulate hypotheses that are verifiable through the observation of empirical proceedings, providing resources for understanding patterns of actions, world views (Weltbilder), structural order and lines of development or trajectories. Ideal types are composed of basic concepts that define a "constructed world of ideas" or "idea constructs" (Gedankenbilder), with a distinct analytical functionality-to compare. Later, ideal types compel researchers to conduct a comparison between the constructed ideal type and the 'historical case.'

The ideal type is a normative and heuristic model or scenario that does not need to emerge from the experiences of a successful case (e.g., developed countries). It does not need to include optimally technical solutions, because it can also refer to a case where conditions are merely tolerated for pragmatic reasons. As George Jellinek (1900) suggests, ideal types can only be manifested as incomplete as they disregard one or more parts of reality, limiting the complex characteristics of the case. The divergence between the ideal type and the historical case serves as leverage point for formulating questions and constituting explanations.

The primary value of ideal types is to enable the formulation of relevant research questions that do not claim the ability to reproduce reality or to predict the future. An ideal type is not the 'perfect situation' or the utopia. While some critics argue that the ideal type tends to focus on extreme phenomena and overlook the connections between them, the ideal type developer can be flexible in designing the ideal type. In this book, the ideal type of the transformation process has been aligned to the stages of the negotiation process and assumes the perspectives of collective decision-making. Nevertheless, ideal types should also allow the construction of simple concepts and fictional conceptual extremes 
(Grenzbegriff), which, however, can still occur in reality. Therefore, ideal types can contribute substantially when conceptualizing complex phenomena.

Ideal types are frequently also regarded as merely applying lessons from successful cases to less successful ones. European forms of democracy, governance, welfare systems and even climate protection schemes are frequently regarded as the end of the evolutionary process (Najam 2005; see Beyerlin 2006; Najam 2010), and this optimal stage is nothing else but the ideal type. This is, however, not how Max Weber and Georg Simmel define ideal types or categories. It is merely a methodological instrument of constructing ideas, a Gedankenexperiment. Moreover, as Alexis de Tocqueville (1856) stressed, although institutions or systems may be similar in some details, they cannot be reproduced as each system is unique. Therefore, borrowing lessons from one case to another, for example from a developed country to a developing country, will most likely involve incomplete information. Although there is still merit in looking at the evolutionary dimension of the development of developed countries, this is not the primary value of ideal types as depicted in this book.

\subsection{Case Studies as Historical Analyses-The Value of Context}

As a method of scientific inquiry and research, case studies have long assumed a well-established and prominent status in many academic disciplines (see McKeown 1999; Maiden 2004; George \& Bennett 2007). While it is often thought that Frederic Le Play first introduced the case-study method into social science in 1829 (see Healy 1947), similar forms of case-study methods can be traced to earlier historical times, even to as early as Ancient Greece (see Lloyd 1992). Case study analysis involves a method that uses the up-close, in-depth, and detailed examination of an object of study (the case) in a specific spatial, temporal, cultural, social, political, and economic context. The usage of case studies in social science has often been chosen, particularly because of its descriptive value in explaining the outcomes or effects of variables. Nevertheless, the systematic further development of case study methods for the cumulative building of theories from various disciplines of social science is often neglected (see George \& Bennett 2007).

A major point of debate on the viability or scientific validity of case study analyses as methods of scientific inquiry is the capability of formulating generalizations from single cases and applying these generalizations to other cases. Karl Popper (1959) has suggested the use of falsification or falsifiability, which forms part of critical reflexivity, as a test to determine the generalizability of 
the outcomes of case studies. He claimed that falsifiability defines the boundary between the scientific and unscientific, whereas that which is unfalsifiable is classified as unscientific. Based on this definition, the possibility that the outcomes of a case study are not applicable to other cases makes case study methods scientific.

\subsubsection{Grasping Historicity and Plurality Through Case Studies-The Value of Perspectives in Knowledge Generation}

What makes case studies interesting for this book is that it highlights the value of perspectives in generating, testing, re-testing, validating, rejecting and accepting knowledge. The use of perspectives in scientific inquiry ensures the acceptance of the pluralism of ideas, actions, normative systems and even of the concepts or understandings of fairness and justice. Such perspectives automatically provide a foundation for revisiting and the eventual modification of ontological concepts. While case study methods reiterate the uniqueness of each case, they are still able to identify and conceptualize patterns, as overlaps, cross-cuts and deviations contribute additional resources for further theory development.

Case studies of transformation processes highlight the relevance of historicity and plurality in understanding the process. Because each transformation process is unique, historicity can only be understood through the perspectives of actors, issues, and structures. Some scholars may even argue that when looking at the perspective of power (power-centric), interactions between actors can be explained and predicted. Perspectives can even define the sources and value of power. The case study approach takes a snapshot of a historical episode, assesses the interactions of various variables and comes up with historical explanations that can be used to explain other events, which could have happened in the past or the future as well as in other geographical areas.

As discussed in the introduction of this book, time is a central pillar in any transformation process. Case studies attempt to provide an account of relevant turning points, lock-ins, set-backs and leapfrogging moments that have occurred. A case study can only grasp one set of these events. Therefore, case studies are based on the presumption that there is a plurality of turning points, lock-ins, set-backs and leapfrogging moments, which are particularly dependent on perspectives. In order words, case studies most likely come from a specific perspective, depending on the preferences and purposes of the chosen actors and frameworks. 


\subsubsection{Procedures and the Design of Case Studies-The Role of Theoretical Models in Case Studies}

The value of comparing case studies among themselves and comparing these case studies with theoretical models, here presented as ideal types, is measured by how these case studies and theoretical models complement each other and how differences can be understood and explained. In addition, the value of comparing case studies and ideal types can be measured by how complex contingent generalizations can be formulated to support decision-making.

In this book, looking for the evident complementarity of case studies and the ideal types will require the following assumptions:

- Ideal types serving as formal models and scenarios have storylines and concepts that can be tested using the case studies.

- Case studies examine how and why these cases overlap and/or deviate, while identifying the new variables or new interdependencies that are responsible for the deviance. As Alexander George and Andrew Bennett (2007) suggest, deviant or outlier cases may be particularly useful for heuristic purposes, as by definition their outcomes are not what traditional theories would anticipate. In addition, the ability to explain this deviance enriches theories, as they suggest new terrains that need to be further understood and explained.

- Case studies evaluate the causal mechanisms behind the correlations or patterns between variables that have been assumed by current theories. Case studies provide a controlling mechanism to check whether correlations are spurious or potentially causal, adding insights into how causal mechanisms can be newly understood as well as how new variables or the refinement of concepts can be specified and operated.

To ensure coherence in the chapters on case studies, the following design and procedures will be implemented:

\subsubsection{Problem and Objectives}

Each case study of this book aims to introduce a selection of the most important subjects and objects of analysis that will be used to examine the specificities of the transformation process. While the subject of the case study refers to the promotion or obstruction of the low-carbon transformation process, the following will be taken as objects of the case study analysis: 
- Recent developments, particularly how the country refers to the Paris agreement and the Sustainable Development Goals (SDGs).

- The multiple entry points to $\mathrm{SD}$, particularly climate mitigation and energy security

- The multiple goal-setting of policies through different scales and levels

- Challenges, barriers, trade-offs, caveats, gaps and risks

- Opportunities, synergies, and co-benefits

- System transition and/or transformation (multiple transitions) including socio-economic, cultural, technological, political, and demographic drivers and trends of change (e.g., GDP, population, migration, R\&D)

- Path dependence and lock-ins: infrastructure, governance, behavioral aspects

- Enabling or hindering frameworks for behavioral and lifestyle change: policy instruments, non-mitigation policies, non-SD policies, state partnerships with private sector and civil society

- International cooperation and institutions

- Legitimacy: transparency, accountability and social inclusion in decision-making

- Innovation and technology development, diffusion and transfer

The analysis of these subjects will allow the inductive identification of new variables, hypotheses, causal mechanisms and causal paths that are needed to be understood in order to correct the paths or benchmarks of low-carbon transformation. In addition, the discovery of potential causal relationships may initiate new methods of quantitative and qualitative measurement.

\subsubsection{The Execution of the Case Studies}

The execution of the case studies as methods of scientific inquiry will be conducted through the following steps:

1. The formulation of the chronological 'narrative' that defines each chapter

2. The identification and assessment of causal mechanisms through congruence methods and process-tracing

3. A deviation analysis between the empirical case studies and the ideal types

4. A Comparative analysis of two selected (country) case studies (where applicable)

For each chapter, accessible primary literature, secondary literature and interview data will be used to construct a chronological 'narrative' that will structure historical inquiry. Such an inquiry aims to establish the values of independent and dependent variables. 
In addition, the case studies aim to highlight the causal mechanisms that are substantial in promoting or obstructing low-carbon transformation. Looking at particular cases, it is argued that such causal mechanisms can operate only under certain conditions and that their effects are highly contingent upon interactions between factors. As Alexander George and Andrew Bennett (2007) claim, a causal mechanism may be necessary, but is not sufficient, in an explanation. In other words, effects or occurrences are brought about by a set or bundle of factors and configurations of mechanisms, through which the behavior of actors is defined. Nevertheless, the same bundle may constitute other effects or even counteract the effects when transferred to another country or even region within the same country. Therefore, one causal mechanism will be compared with another case study in the chapter as well as compared with the theoretical model (ideal type) to analyze how a causal mechanism offsets other causalities in some contexts and complements them in others (see George \& Bennett 2007).

\subsubsection{Congruence Methods-The Role of Process Tracing}

The relevant theories are summarized through the ideal types. Linking these theoretical models to the case studies is an attempt to assess the ability of these theories to explain or predict the occurrence of events or factors. As Alexander George and George Bennett (2007) suggest, if the outcome of the case studies is consistent with the predictions of the theories, then there is the possibility that a causal relationship exists. Overlaps and cross-cuts can explain the applicability of theories to realities. In cases of deviations, explanations will be sought for these deviations, and hypotheses will be formulated about how these deviations will need to expand the existing theories. In cases of both congruence and deviation, factors and theoretical reasons will be taken into consideration to address how the hypothesized causes may be amplified, diminished, delayed or sped up (e.g., the self-fulfilling prophecy).

The next step pertains to the assessment of the possible causal significance of congruity (see George \& Bennett 2007), where two questions will be asked:

- Is the consistency spurious or of possible causal significance?

- Is the independent variable a necessary condition for the outcome of the dependent variable and how much explanatory or predictive power does it have?

As suggested by Alexander George and Andrew Bennett (2007), spuriousness occurs when the observed congruence of the cause $\mathrm{C}$ and effect $\mathrm{E}$ is artificial because both $\mathrm{C}$ and $\mathrm{E}$ are caused by some third factor $\mathrm{Z}$. 


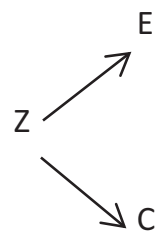

Alternatively, the putative cause $\mathrm{C}$ lacks causal priority if $\mathrm{C}$ is necessary for $\mathrm{E}$, but $\mathrm{C}$ is itself only an intervening variable wholly or largely caused by a necessary prior variable $\mathrm{Z}$. In this instance, both $\mathrm{Z}$ and $\mathrm{C}$ are necessary for $\mathrm{E}$, but $\mathrm{C}$ has no independent explanatory value. The comparability of these country cases will be supported by the general questions formulated that link these subjects of analysis (see above) to the low carbon transformation process. The outcomes of the case studies will be explained by referring to the theoretical models (ideal types). Deviations will then be highlighted while providing theoretical and empirical explanations for these deviations. In many cases, the theoretical framework provided by the ideal types will need to be broadened to capture the major aspects of the historical elements. This broadening defines the analytical value of these case studies by allowing typological theorization.

To summarize, process tracing is a method that motivates one to take equifinality into account. This refers to an openness to the possible feasibility of alternative paths through which the outcome could have occurred. In addition, process tracing supports the possibility of mapping out one or more potential causal paths that are consistent with the outcome. As other cases are identified and tested, new typologies can be developed, leading to the further development of existing theories.

\subsubsection{Theory Testing and the Implications of Case Findings for Theory and Practice}

An analysis through case studies pertains to the testing of deductive theories. Theory-testing aims to strengthen or reduce support for a theory, narrow or extend the scope conditions or parameters of a theory, or determine which of two or more theories can best explain a case, type or general phenomenon (George \& Bennett 2007 p. 112). As theories are tested using the data and information from the case studies, this testing may lead to an assessment that existing theories are not able to provide the necessary framework of understanding. Therefore, case studies can also initiate an inductive process of theory generation. 
An analysis of deviations from theoretical models (ideal types) can link the gaps between deductive and inductive processes. As case studies uncover new or omitted variables, self-driving dynamics, hypotheses, causal paths, causal mechanisms, types of interaction, and network effects, the historical explanation that has been supported by existing theories can be improved upon (see George \& Bennett 2007). This improvement highlights previously overlooked variables. In some cases, some of these new factors can be a highly significant factor that delays or obstructs the transformation process.

Developing or expanding upon existing theories through the analysis of case studies is an inductive process. Single case studies can produce observations that can initiate further questions, detect patterns (which can be supported by additional case studies as well as by other forms of scientific inquiry such as experiments), and hypothesize on tentative explanations of these observations. Hypothesizing can include distinguishing the subclasses or subtypes of existing typologies. In addition, 'concatenated' theories can be developed by dividing complex causal processes into specific component theories, or sequential stages, phases or episodes, as well by as focusing on the perspectives of identified actors (see George \& Bennett 2007). For example, by focusing on actors' behavior and how it changes as the process unfolds will provide important insights into sequential stages and typical interactions and dynamics within a specific stage or episode.

As the deviance between the theoretical model and the case studies becomes clear and eventually leads to the specification of a new theory, generalizations can be achieved as to how the newly identified mechanism may play out in different contexts and how this new mechanism can be relevant in managing processes (George \& Bennett 2007 p. 112).

\subsection{Role-Play Simulation}

Simulation as a method of scientific inquiry gained popularity in the 1990s as computer simulations were used to simplify and mimic complex systems. Considered the third pillar of empirical research, along with experiment and theory (see Drösser 1994), simulations are recognized as allowing the manipulation of complex systems that would be impossible, too costly or unethical to do so in natural systems (Peck 2004). Simulation can add to theory development and testing, can assist in formulating hypotheses and can limit the scope of which data are most important to gather (see Peck 2004; Gilbert \& Troitzsch 2005). 
This book does not intend to do computer simulations, but rather a so-called 'role-play' simulation. Particularly because this book highlights the aspects pertaining to functional, institutional, and bargaining interactions, a simulation was conducted using the role-pay format, where the participants were brought together to mimic interactions under conditions and parameters set by the game designer. For simplicity, when referring to simulations in this book, role-play simulations will be meant. In this book, more than 15 postgraduate students from the University of Technology in Kingston, Jamaica, participated in a role-play simulation. While the main goal of the simulation was educational, the role-play simulation was also designed to provide useful empirical insights into the analysis of sustainable, low-carbon transformation.

\subsubsection{The Scientific Value of Role-Play Simulations}

While the pedagogical value of role-play simulations in the classroom is less contentious, the value of the results from these games to scholarly research may require further discussion, if not further convincing of their scientific value. The engagement students as subject groups or 'human guinea pigs' may be perceived as self-serving and drawing empirical evidence from these games may be limited. Furthermore, most of the simulation games used in classes of international relations and political sciences depict complex cases such as climate change negotiations, where negotiators are required to have extensive institutional memory and technical expertise. While role-play simulations will mostly run for mere hours, these real-life negotiations occur for a longer period of time, with several preparatory meetings and consultations.

Nevertheless, the limitations of the empirical value of role-play simulations are not limitations per se, because there are distinct expectations and none of these expectations is to reproduce reality. Like computer simulations and models, role-play simulations aim to look at specific aspects of negotiations. Variables and parameters are adjusted to look at how processes are affected by such changes. In addition, particularly for this book, role-play simulations are used to identify interesting impulses that will then become subjects of further scientific inquiries. Moreover, some events in the role-play simulations are compared to the real-world and some theories and empirical statistics are drawn to explain differences. These explanations will inevitably test the relevance of existing theories and suggest improvement or even encourage the development of new hypotheses for further research. Therefore, in any case, role-play simulations can in actuality promote further theory development. 
The purpose of conducting simulations as a method of scientific inquiry is to introduce the possibility of a new way of thinking about social processes that is difficult to understand through text books (Penetrante 2012). Based on ideas about the emergence of the complex mechanisms of interactions between actors, a holistic and meta-level analysis is made possible. Because simulation is akin to an experimental methodology, simulation can be executed many times to draw conclusions through analogies (Müller 1998). The variation of conditions through the researcher allows the exploration of the several effects of different parameters (Gilbert \& Troitzsch 2005 p. 14).

\subsubsection{The Procedures and Design of the Role-Play Simulation}

This book puts forward the procedures and design of role-play simulations that highlight the process and negotiation outlooks on a complex system. As mentioned earlier, in role-play simulations, such as those conducted in this book, the dynamics of functional, institutional, persuasive and bargaining interactions initially depend on the parameters preconditioned by the researcher, who will define the initial point of behavior, based on assumptions made prior to the game. These assumptions are to be clearly communicated to allow the participants to understand the scope of the issues that are relevant to the game.

These assumptions can be formulated through the following steps:

First, the role-play designer chooses an aspect of a complex system. For example, in chapter 12, a cabinet meeting of the Jamaican national government on budget-planning under the conditions set by the International Monetary Fund.

Second, the role-play designer identifies the problems and challenges he or she wants to be understood through the game. The problems and challenges will define the agenda of the role-play game and will limit the scope of parameters. A game can be limited to a single problem. In chapter 12, the difficulty of consensus-building in achieving the budget plan will be presented as the over-arching problem. In this game, the agenda becomes clear, which helps structure the discussions of the participants. In addition, a game can also highlight several problems and challenges. Here, more than one problem was introduced, and these need to be resolved in parallel, in conjunction or in a specific order by the participants. In addition, each interacting participant has a different set of problems and challenges, where resolving them requires cooperation with other participants who may not have the same set of problems. 
Third, questions need to be formulated that will identify the parameters that will be manipulated. For example, in chapter 12, one question refers to the effects of gender. In one group, all the members were females, in another all male, and in the other mixed. In addition, the parameters can be addressed by introducing control and experimental groups to allow direct comparisons.

Fourth, the profiles of participants need to be prepared and distributed among the participants before the games. These profiles need to include brief background information about the positions and interests of the country. These positions and interests are to be concretized in specific numbers (e.g., 20 million US dollars as compensation; or maximum 10 percent in GHG emissions by 2030). Each profile also needs to clarify the limitations of the negotiators. For example, a 'toxic' issue can be identified, where negotiators would need to walk away if this issue is involved. The profiles also include some instructions or recommendations as to how they should behave at the bargaining table: competitive, collaborative, avoidant, cooperative, emotional, arrogant, etc. to highlight the human factor at negotiations.

Fifth, the role-play designer needs to plan the departure point of the game. It may include requiring all participants to give a brief (ministerial) statement in a plenary session or to instruct each participant which bilateral or mini-lateral meeting he or she needs to prioritize first. These brief ministerial statements allow the participants to have an overview of potential partners and adversaries. This 'departure point' also serves as an 'ice-breaker', as participants will eventually 'warm up' during the game.

Sixth, some flexibility and creativity should be encouraged in the game to allow the participants to come up with innovative and out-of-the-box solutions. Furthermore, some provisions should be made leading to changes, for example, in the power or interests of one or more participants. In addition, while the simulation is running, the participants may choose to follow instructions and adapt to these parameters and frameworks, basing their decisions on certain rationalities and criteria. With the researcher intentionally changing the parameters and frameworks as the game unfolds, the effects of such changes should be observed, documented and discussed during debriefings. These observations would generate (simulated) data, which can be later compared to the data produced by other methodologies outside of the simulation (Penetrante 2012).

Seventh, the results of the role-play should be clear and concrete, such as forging an agreement with one or more counterparts, or 'sabotaging' and 'stalling' the negotiations. This allows closure in the game. Furthermore, the role-play designer needs to carefully plan the evaluation discussion and debriefing to encourage the participants to reflect on what they have experienced. The participants 
should also be asked about their feelings during the game, whether their expectations are met, whether they would like to change something about how they negotiated were they given the chance, or what struck them most during the game. Also, technical and logistical questions about the game should be encouraged. Are there some shortcomings in the game and whether there are things they would like to change about how the game was designed and conducted?

Eighth (and finally), participants should be given a questionnaire or a written task that they need to do in few days after the experience has sunk in. It should be submitted no longer than a week after the game. This questionnaire with both open and closed questions could include questions that can be useful for the researcher when assessing the empirical value of the results.

\subsection{Interim Conclusion: Bridging Gaps Between Theory and Practice Through Knowledge and Decision Tools}

The knowledge and decisions tools introduced in this chapter can be particularly helpful in identifying and assessing obvious and latent stumbling blocks to effective collective decision-making. These tools can also provide impulses for how these stumbling blocks can be addressed to allow actors to cope with them. Major challenges and barriers to low-carbon system transformation are diverse. The first step of bridging the gaps between theory and practice is to ensure the fluidity of the relationship between the two. Theory generation should be dynamic, as reality becomes more complex with the emergence of new issues that require multi- and interdisciplinary as well as an integrated outlook to grasp their essence and meaning. As challenges and barriers are identified by policy-makers (covered here by the case studies) and academes (covered by the theoretical models), their perspectives combine, and new insights can be found that can allow more effective measures to facilitate the transformation process.

It is the aim of this book that the theoretical models (ideal types), case studies and the role-play simulations will contribute to an understanding of how gaps between theory and practice can be bridged. The case studies and role-play simulations will be conducted to understand how low-carbon transformation processes can be facilitated. What kind of theoretical and practical knowledge is needed by decision-makers for dealing with challenges, risks, caveats and barriers related to sustainable, low-carbon transformation? How can these case studies and role-play simulations contribute to the establishment of general, conceptual models that can be helpful in designing and implementing policy instruments? 
Open Access This chapter is licensed under the terms of the Creative Commons Attribution 4.0 International License (http://creativecommons.org/licenses/by/4.0/), which permits use, sharing, adaptation, distribution and reproduction in any medium or format, as long as you give appropriate credit to the original author(s) and the source, provide a link to the Creative Commons license and indicate if changes were made.

The images or other third party material in this chapter are included in the chapter's Creative Commons license, unless indicated otherwise in a credit line to the material. If material is not included in the chapter's Creative Commons license and your intended use is not permitted by statutory regulation or exceeds the permitted use, you will need to obtain permission directly from the copyright holder.

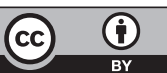

